\title{
Mobile Ion-Driven Modulation of Electronic Conductivity Explains Long-Timescale Electrical Response in Lead lodide Perovskite Thick Pellets
}

\author{
Marisé García-Batlle, Sarah Deumel, Judith E. Huerdler, Sandro F. Tedde, Antonio Guerrero, \\ Osbel Almora, and Germà Garcia-Belmonte*
}

Cite This: ACS Appl. Mater. Interfaces 2021, 13, 35617-35624

Read Online

ACCESS | Lلll Metrics \& More | 回 Article Recommendations

Supporting Information

ABSTRACT: The favorable optoelectronic properties of metal halide perovskites have been used for X-and $\gamma$-ray detection, solar energy, and optoelectronics. Large electronic mobility, reduced recombination losses of the electron-hole pairs, and high sensitivity upon ionizing irradiation have fostered great attention on technological realizations. Nevertheless, the recognized mixed ionic-electronic transport properties of hybrid perovskites possess severe limitations as far as long-timescale instabilities and degradation issues are faced. Several effects are attributed to the presence of mobile ions such as shielding of the internal electrical field upon biasing and chemical interaction between intrinsic moving defects and electrode materials. Ion-originated modu-
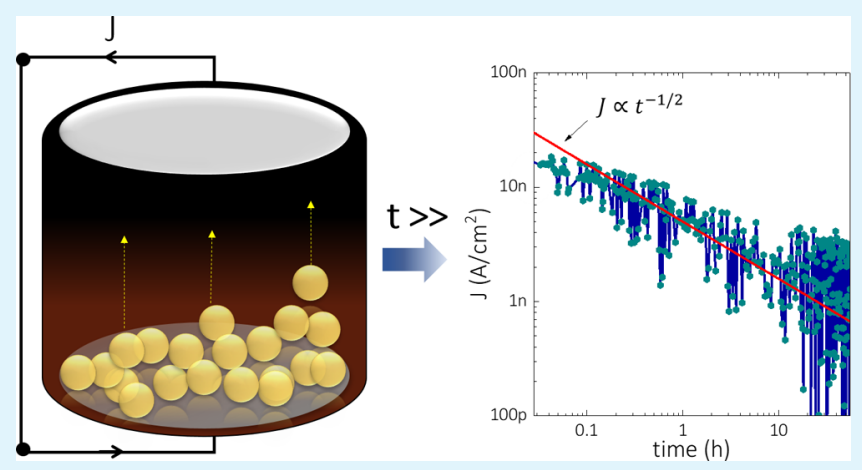
lations of electronic properties constitute an essential peace of knowledge to further progress into the halide perovskite device physics and operating modes. Here, ionic current and electronic impedance of lead methylammonium iodide perovskite thick pellets are independently monitored, showing self-consistent patterns. Our findings point to a coupling of ionic and electronic properties as a dynamic doping effect caused by moving ions that act as mobile dopants. The electronic doping profile changes within the bulk as a function of the actual ion inner distribution, then producing a specific time dependence in the electronic conductivity that reproduces time patterns of the type $\propto \sqrt{t}$, a clear fingerprint of diffusive transport. Values for the iodine-related defect diffusivity in the range of $D_{\text {ion }} \sim 10^{-8} \mathrm{~cm}^{2} \mathrm{~s}^{-1}$, which corresponds to ionic mobilities of about $\mu_{\text {ion }} \sim 10^{-6} \mathrm{~cm}^{2} \mathrm{~V}^{-1} \mathrm{~s}^{-1}$, are encountered. Technological realizations based on thick perovskite layers would benefit from this fundamental information, as far as long-timescale current stabilization is concerned.

KEYWORDS: perovskites, ion migration, electronic conductivity, dynamic doping, X-ray detection

\section{INTRODUCTION}

The significant success achieved by hybrid organic-inorganic lead halide perovskites is attributed to their outstanding light harvesting and charge carrier collection properties. This has motivated intensive research for photovoltaic applications ${ }^{1}$ and, among other subjects, for high-energy radiation detectors and imaging devices for medical diagnostics. For instance, it is well known that $\mathrm{CH}_{3} \mathrm{NH}_{3} \mathrm{PbI}_{3}$ (MAPI) shows promising sensitivity for ionizing radiation detection with competitive values compared to standard technologies. ${ }^{2}$ Similarly, the favorable optoelectronic properties of similar perovskites have been widely studied for X-and $\gamma$-ray detection, and complete devices have been proved. ${ }^{3,4}$ Current realizations of perovskitebased radiation detectors are typically made of bulky devices with absorber thicknesses up to millimeters to stop high-energy $\mathrm{X}$-ray photons, poly-to-monocrystalline morphology, and asymmetric metallic contacts. ${ }^{5-7}$ These structural features differ from those encountered in thin-film perovskite photo- voltaics, where much is currently known about the electronic characterization and modeling of the charge carrier transport properties. ${ }^{8,9}$ Nevertheless, the mixed ionic-electronic transport properties of hybrid perovskites are still a puzzling subject of research either for solar or ionizing radiation devices. Moreover, there are not too many studies on the longtimescale electric response of ionizing energy detectors based on thick perovskite layers ${ }^{3}$ and further understanding of their working mechanisms is certainly needed. ${ }^{10}$

Classical modeling of semiconductor devices considers mobile electronic carriers while includes the influence of

Received: April 1, 2021

Accepted: July 14, 2021

Published: July 26, 2021 
fixed ionic defects determining the electronic doping profile. Usually, transport properties of ionic species play a minor role in the overall short-term operation. However, drift and diffusion of dopants ${ }^{11,12}$ or intrinsic defects ${ }^{13,14}$ constitute well-known degradation mechanisms in traditional solar cells and other electronic devices. Degradation typically occurs during long-term operational conditions, and the involved ionic species come either from electrodes, or intermediate layers in heterostructures, or result from the product of the reactivity of the materials. Importantly, the mobility $\mu_{\text {ion }}$ of these ionic species in traditional semiconductor materials is many orders of magnitude lower than typical values $\left(\mu_{\mathrm{el}} \sim 100\right.$ $\mathrm{cm}^{2} \mathrm{~V}^{-1} \mathrm{~s}^{-1}$ ) of the electronic mobility.

As an example of an optoelectronic device in which the interplay between electronic and ionic kinetic properties plays a determining role, we can cite light-emitting electrochemical cells. ${ }^{15}$ Here, the ionic charge dynamics is essential to explain the time evolution of the light power emission as these materials are mixed ionic-electronic conductors. ${ }^{16}$ For this kind of disordered organic devices, electronic mobilities are intrinsically lower $\left(\mu_{\mathrm{el}} \sim 10^{-5} \mathrm{~cm}^{2} \mathrm{~V}^{-1} \mathrm{~s}^{-1}\right)$ because of the polaronic character of the carrier transport in amorphous organic films, ${ }^{17}$ approaching the expected ion mobilities $\mu_{\text {ion }}$ of $\sim 10^{-8}$ to $10^{-5} \mathrm{~cm}^{2} \mathrm{~V}^{-1} \mathrm{~s}^{-1}$ in these materials. ${ }^{15}$ Also, oxygenvacancy transport in oxides ${ }^{18}$ is known to play a determining role in memristive devices to tailor electrical resistance for data storage and neuromorphic applications. ${ }^{19}$

For halide perovskite compounds, the dual ionic-electronic character of charge transport complicates the device modeling and forces the consideration of both faster and slower mechanisms, related to electronic and ionic kinetics, respectively. ${ }^{20,21}$ It is known that several intrinsic defects (vacancies and interstitials as $\mathrm{V}_{\mathrm{I}}^{+}, \mathrm{MA}_{i}^{+}, \mathrm{V}_{\mathrm{MA}}^{-}$, or $\mathrm{I}_{i}^{-}$in the case of MAPI $)^{22,23}$ possess non-negligible transport properties. ${ }^{24,25}$ Several effects are attributed to the presence of mobile ions such as alteration of the internal electrical field upon biasing, through contact charge accumulation and shielding. ${ }^{26-28}$ The chemical interaction between intrinsic moving defects and electrode materials giving rise to losses in performance and device instabilities is also reported. ${ }^{29,30}$

Moreover, the electronic and ionic dynamics relates through coupling of either recombination or conduction electronic carrier properties with defect distribution within the active layer. In this line, it was suggested for perovskite solar cells that carrier recombination flux is highly dependent on the local ionic environment, in such a way that upon operation, ions move to reduce recombination resistance, slowing down the nonradiative recombination rate. ${ }^{31,32}$ Also, the change in doping profile upon poling has been revealed by means of Mott-Schottky analysis, signaling an interplay between ion distribution and electronic density. ${ }^{33}$ Even more interesting is the observation of electronic conductivity modulation in connection with ionic migration. For example, the time dependence of the electrical current in MAPI pellets with planar symmetrical electrodes has been correlated with the drift of the photoluminescence front upon biasing. The effect is explained by a sort of ion-induced modification of the doping profile in which the drift of vacancies establishes the local electron and hole concentration and consequently the steadystate electronic conductivity properties. ${ }^{34}$ In other cases, ion diffusion, instead of drift, has been claimed as a suitable explanation for the impedance relaxation at zero bias. ${ }^{35}$ This last approach has permitted the estimation of the ion diffusion coefficient $\left(D_{\text {ion }} \sim 10^{-8} \mathrm{~cm}^{2} \mathrm{~s}^{-1}\right)$ in thick lead bromide perovskite single crystals.

Hence, the coupling between ionic and electronic properties in halide perovskites is a central issue that has mobilized many efforts and fostered important research during the last years. ${ }^{36,37}$ Although some partial and/or indirect correlations between ionic and electronic mechanisms have been proposed or invoked as explanatory rationales of the device operation, unambiguous observations of these mixed ionic-electronic interplays are still obscure. ${ }^{38-41}$ However, deciphering ionoriginated modulations of electronic properties might constitute an essential piece of knowledge to further progress into the halide perovskite device physics and operating modes. Also, specific technological applications such as solar cells, light-emitting diodes, or X-and $\gamma$-ray detectors would benefit from this fundamental information, particularly when longtimescale functioning and degradation paths become an issue.

In this work, the electrical response of $\sim 1 \mathrm{~mm}$-thick $\mathrm{CH}_{3} \mathrm{NH}_{3} \mathrm{PbI}_{3}$ microcrystalline pellets upon biasing is explored by registering current transients and impedance spectra. Several long-time experiments have been programmed that observe clear ion diffusion trends in the range of 1 day. Simultaneously, the electronic conductivity is monitored that reproduces the same time patterns of the type $\propto \sqrt{t}$, a clear fingerprint of diffusive transport. Our findings point to couple ionic and electronic properties as a dynamic doping effect caused by moving ions that act as mobile dopants. Ion diffusion after removing bias changes the local doping density, governing as a consequence the electronic transport. This approach allows extracting values for the ion diffusivity in the range of $D_{\text {ion }} \sim 10^{-8} \mathrm{~cm}^{2} \mathrm{~s}^{-1}$, which correspond to ion mobilities of about $\mu_{\text {ion }} \sim 10^{-6} \mathrm{~cm}^{2} \mathrm{~V}^{-1} \mathrm{~s}^{-1}$ by assuming Einstein's relation, in good agreement with other independent measurements (see Table S2 in Supporting Information).

\section{RESULTS AND DISCUSSION}

The samples under consideration were $\sim 1 \mathrm{~mm}$-thick MAPI microcrystalline pellets asymmetrically contacted with evaporated $\mathrm{Pt}$ and $\mathrm{Cr}$ electrodes of $1 \mathrm{~cm}^{2}$ area. The fabrication details, basic morphology, and optoelectronic characterization can be found in Table S1 of Supporting Information. The Experimental Section also summarizes the used instrumentation and methods.

Short-Time Current Transients. We first checked the sample response to voltage steps (inset of Figure $1 \mathrm{~b}$ ). The current transients after applying different positive biases during $500 \mathrm{~s}$ are presented in Figure 1a. The electronic current is delayed during the first $200 \mathrm{~s}$, presumably due to the reordering of the ionic density. ${ }^{42}$ During the transient, the electronic charge carriers may be influenced by the ionic drift, which generates an ionic current with the same polarity as that of the electronic current. Eventually, the ions accumulate near the perovskite-electrode interfaces, increasing the ion-induced built-in electric field and leading to the simultaneous operation of drift and diffusion currents before the ion accumulation reaches the equilibrium condition. ${ }^{43,44}$ Maximum currents upon bias application are registered in the range of $\mu \mathrm{A} \mathrm{cm}$ (Figure 1a). Subsequently, after positive biasing, the device is then kept under short-circuit ( $0 \mathrm{~V}$ bias) conditions to observe the relaxation current, as presented in Figure $1 \mathrm{~b}$.

When the external bias is removed, a small undershoot current appears of the order of $\mathrm{nA} \mathrm{cm}{ }^{-2}$ with the opposite sign (Figures $1 \mathrm{~b}$ and $\mathrm{S} 1 \mathrm{~b}$ ). That negative current is the fingerprint 

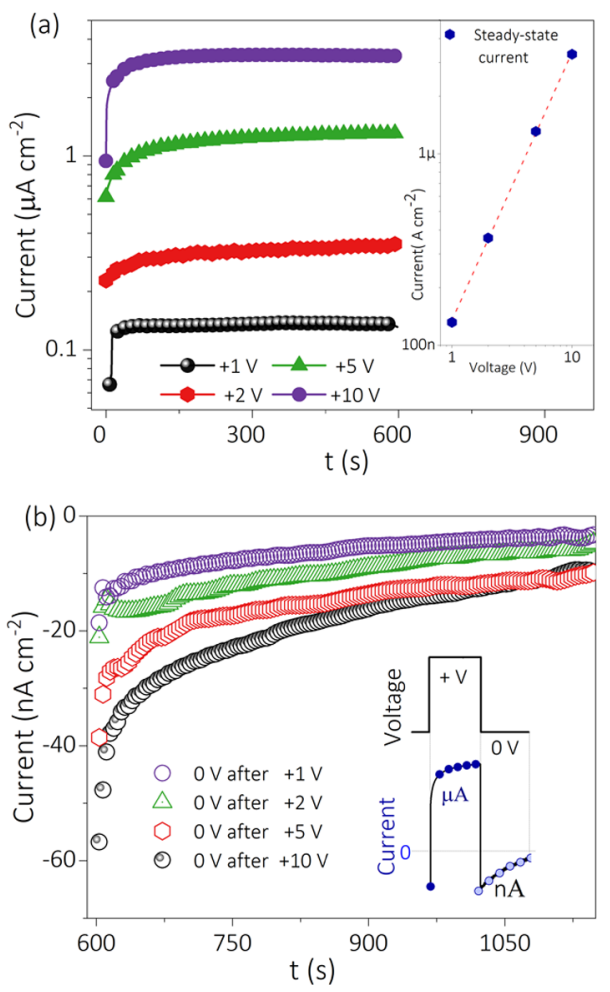

Figure 1. (a) Short-time current transients registered at different positive applied biases. In the inset, there is the corresponding variation of the steady-state current (dots) and fit (dashed line). (b) Relaxation current registered after the positive biasing when the device is kept under short-circuit condition $(0 \mathrm{~V})$. Similar responses are obtained for negative biasing. The inset in (b) schemes the bias step and order of magnitude and sign of the current transients.

of initial ion displacement to recover equilibrium distribution, as remarked previously. ${ }^{42}$ Note that electronic displacement currents should respond faster $(\sim \mu \mathrm{s})$ within the scale of the dielectric relaxation time (see later on this concern). Zero-volt current registering is then a way of observing ionic migration without the perturbing contribution of electronic carriers. It is observed that the ionic current decay occurs in two steps: first, a quick response during a few seconds and afterward, a slower relaxation in a timescale of tens to hundreds of seconds. These processes may correspond to the slow reduction of the ionic built-in field, which drifts backward the ions at the same time that they diffuse to restore equilibrium. ${ }^{42}$ Negative overshoot currents are always situated in the range of $\mathrm{nA} \mathrm{cm}$ (see Figure $1 \mathrm{~b}$ ). The slower transient response can be fitted using an exponential decay to obtain a characteristic time constant of $\tau_{\text {st }} \sim 200 \mathrm{~s}$ (Figure S1b).

One might think about an ionic relaxation process involving long distances within the perovskite thick layers. An estimation of the ion diffusion coefficient easily results using the next expression

$$
D_{\text {ion }}=\frac{L_{\text {diff }}^{2}}{\tau}
$$

where $L_{\text {diff }}=0.55 \mathrm{~mm}$ is the effective diffusion length that here corresponds to half of the layer thickness because of the structural symmetry. By including typical response times in Figure $1 \mathrm{~b}$ into eq 1 , a hypothetical value for the short-timescale diffusion coefficient $D_{\text {ion }}^{\text {st }} \sim 1.5 \times 10^{-5} \mathrm{~cm}^{2} \mathrm{~s}^{-1}$ is obtained. We note that it is far from those reported for common solid-state ionic conductors, $D_{\text {ion }} \sim 10^{-12} \mathrm{~cm}^{2} \mathrm{~s}^{-1},{ }^{45-47}$ and even from the previously reported faster ion migration coefficient $D_{\text {ion }} \sim 10^{-8}$ to $10^{-9} \mathrm{~cm}^{2} \mathrm{~s}^{-1}$ in lead halide perovskites. ${ }^{35,48,49}$ Similarly, Einstein's equation can be used to deduce a characteristic mobility as

$$
\mu_{\text {ion }}=\frac{q D_{\text {ion }}}{k_{\mathrm{B}} T}
$$

where $q$ is the elementary charge, $k_{\mathrm{B}}$ stands for the Boltzmann constant, and $T$ is the absolute temperature. From eq 2, the previous estimation would entail $\mu_{\mathrm{ion}}^{\mathrm{st}} \sim 6 \times 10^{-4} \mathrm{~cm}^{2} \mathrm{~V}^{-1} \mathrm{~s}^{-1}$, which is far above previous determinations of ionic mobility in MAPI $^{8,50}$ (see Table S2). Consequently, the unphysical inferred values of $D_{\text {ion }}^{\text {st }}$ and $\mu_{\text {ion }}^{\text {st }}$ indicate that the characteristic timescale of $\tau_{\text {st }} \sim 200 \mathrm{~s}$ should involve ionic displacements confined within much narrow regions. For this timescale, diffusion within the pellet competes with the remaining effects of the ionic built-in field, which governs the early stages of the relaxation toward equilibrium.

Long-Time Current Transients. The abovementioned discussion suggests that long-range ionic diffusion would require a longer timescale at short-circuit $(0 \mathrm{~V}$ bias $)$, where the drift component due to the ionic built-in field can be neglected. Only then, the decay time constants can reflect the dynamics of the diffusive ionic transport within the whole layer thickness. ${ }^{35,42}$ Therefore, an experiment similar to that previously schemed in the inset of Figure $1 \mathrm{~b}$ was performed in a timescale of days, as illustrated in Figure 2. Replicas of this experiment are shown in Figure S2.

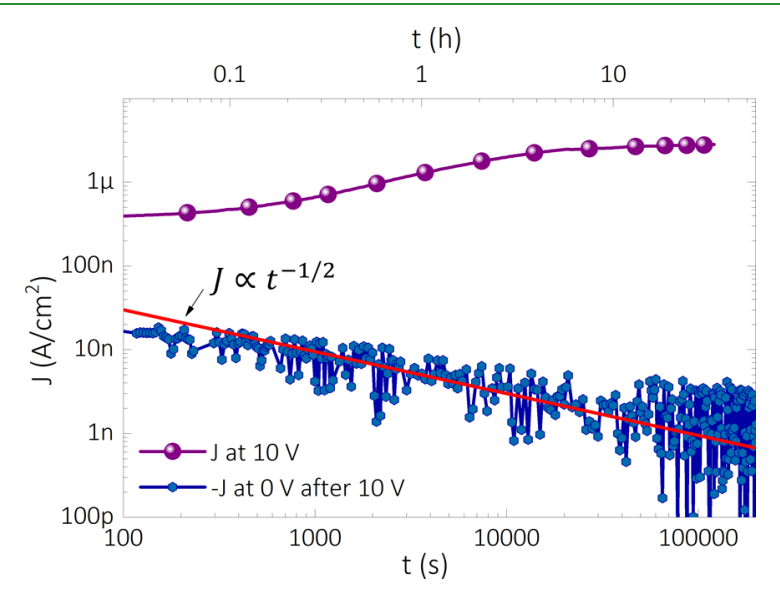

Figure 2. Long-time current transients of MAPI pellets in the dark, under the applied external $10 \mathrm{~V}$ bias and subsequent zero bias, as indicated. The solid red line corresponds to a fit to a $1 / \sqrt{t}$ decay law.

The current during forward biasing was recorded during approximately 1 day, before switching to short-circuit conditions, when the current was measured for around 2 days. During the polarization at $+10 \mathrm{~V}$, an increase in the drift current is observed which seems to saturate at $t \gg 5 \times 10^{4} \mathrm{~s}$. Subsequently, after removing the applied bias, a nondiffusing regime can be first observed for $t<400 \mathrm{~s}$, preceding a clear 1/ $\sqrt{t}$ law for $t \gg 400 \mathrm{~s}$. It is well known that time dependences of the type $\sqrt{t}$ can be taken as an indication for the occurrence of diffusion mechanisms. ${ }^{48}$ As observed in Figure 2, the small ionic current is masked by a noisy component that dominates at longer times $t>5 \times 10^{4} \mathrm{~s}$. Despite the noise of order $\sim 1 \mathrm{nA}$ $\mathrm{cm}^{-2}$, the diffusive component is clearly identified. Unfortu- 
nately, the reduced signal-to-noise ratio precludes reproducing these experiments using lower bias values.

The observed diffusive long-term current decrease can be assumed to have a characteristic ionic diffusion time $\tau_{\text {lt }} \geq 5 \times$ $10^{4} \mathrm{~s}$. Thus, using eqs 1 and 2, one can estimate an upper limit for the long-timescale ionic diffusion coefficient and mobility, which are on the order of $D_{\text {ion }}^{\mathrm{lt}} \sim 10^{-8} \mathrm{~cm}^{2} \mathrm{~s}^{-1}$ and $\mu_{\text {ion }}^{\mathrm{lt}} \sim 10^{-6}$ $\mathrm{cm} \mathrm{V} \mathrm{V}^{-1} \mathrm{~s}^{-1}$, respectively. These values are evidently closer to those previously reported, ${ }^{35,48,49}$ suggesting that the ionic current transient observed in Figure 2 is a fingerprint of ionic diffusion mechanisms involving the entire pellet's bulk.

Long-Time Impedance Recovery. The previous analysis relies on monitoring small currents $\left(1-10 \mathrm{nA} \mathrm{cm}{ }^{-2}\right)$, poorly distinguishable from noise at longer times. An experimental way of augmenting the signal-to-noise ratio consists of measuring the current response to a perturbative stimulus at several stages during the system relaxation at zero bias. This is can be carried out the measurement of the impedance spectroscopy (IS) response of the MAPI thick pellets (in the frequency domain within $10^{-2} \mathrm{~Hz}<f<10^{6} \mathrm{~Hz}$ ), where a small voltage alternating-current $(\mathrm{AC})$ mode perturbation $(20 \mathrm{mV})$ is superimposed to the direct-current (DC) mode bias $(0 \mathrm{~V})$. Because of the small AC and DC values, the experiment is always carried out within the ohmic electronic response regime. ${ }^{10}$ Given that each impedance spectrum takes about $900 \mathrm{~s}$ to be registered, this procedure is a manner of exploring the electronic conduction state of the active layer at different times. By examining Figure 3a, one can observe typical impedance spectra of perovskite-based devices, which comprise a resistor-capacitor $(R C)$-coupled subcircuit at higher frequencies. As discussed previously, ${ }^{35}$ these circuit elements represent the geometrical (dielectric) capacitance $C_{\mathrm{g}}=\varepsilon \varepsilon_{0} A / L$ and the electronic resistance $R=L / \sigma_{\mathrm{el}} A$, where $A$ is the active area, $\varepsilon$ is the dielectric constant, $\varepsilon_{0}$ is the vacuum permittivity, and $\sigma_{\mathrm{el}}$ accounts for the electronic conductivity. It is worth noting that the characteristic response time for this $R C_{\mathrm{g}}$ circuit always lies within those values typical for electronic dielectric relaxation timescales ${ }^{51} \tau_{\text {die }} \sim 10 \mu \mathrm{s}$, reinforcing the electronic origin of the high-frequency impedance response. The whole spectra are analyzed and fitted in terms of the equivalent circuit given in Figure S3.

A detailed view of the high-frequency resistance variation at zero bias for $12 \mathrm{~h}$ after removing $+10 \mathrm{~V}$ bias can be observed in the IS plot of Figure 3a. Once short-circuit ( $0 \mathrm{~V})$ DC conditions are imposed, the samples are able to slowly recover the initial equilibrium. By examining Figure $3 a$, one can infer that the sample resistance is a function not only of the applied bias but also of the poling and recovering time. A closer examination of the variation of the resistance extracted from fitting at zero bias is presented in Figure $3 \mathrm{~b}$. The resistance shows a large initial value $R_{1}$ at shorter recovering times (less electronic conductive state) and progressively reduces to attain a steady-state value $R_{0}$ (background state) after a long equilibration time of $12 \mathrm{~h}$. Such a reduction in bulk resistance is interpreted in terms of the increase in electronic carrier density $n$, which relates to the electronic conductivity as $\sigma_{\mathrm{el}}=$ $q \mu_{\mathrm{el}} n$. The limiting resistances $R_{1}$ and $R_{0}$ give rise to an increase in carrier density from $n_{1}=9 \times 10^{8} \mathrm{~cm}^{-3}$ up to $n_{0}=7 \times 10^{9}$ $\mathrm{cm}^{-3}$, by assuming typical values for the electronic mobility as $\mu_{\mathrm{el}}=10 \mathrm{~cm}^{2} \mathrm{~V}^{-1} \mathrm{~s}^{-1}$. Similar behavior is observed for negative biasing (see Figure S4).

Our findings move us to consider that the redistribution of ionic species along the layer bulk is accompanied by the
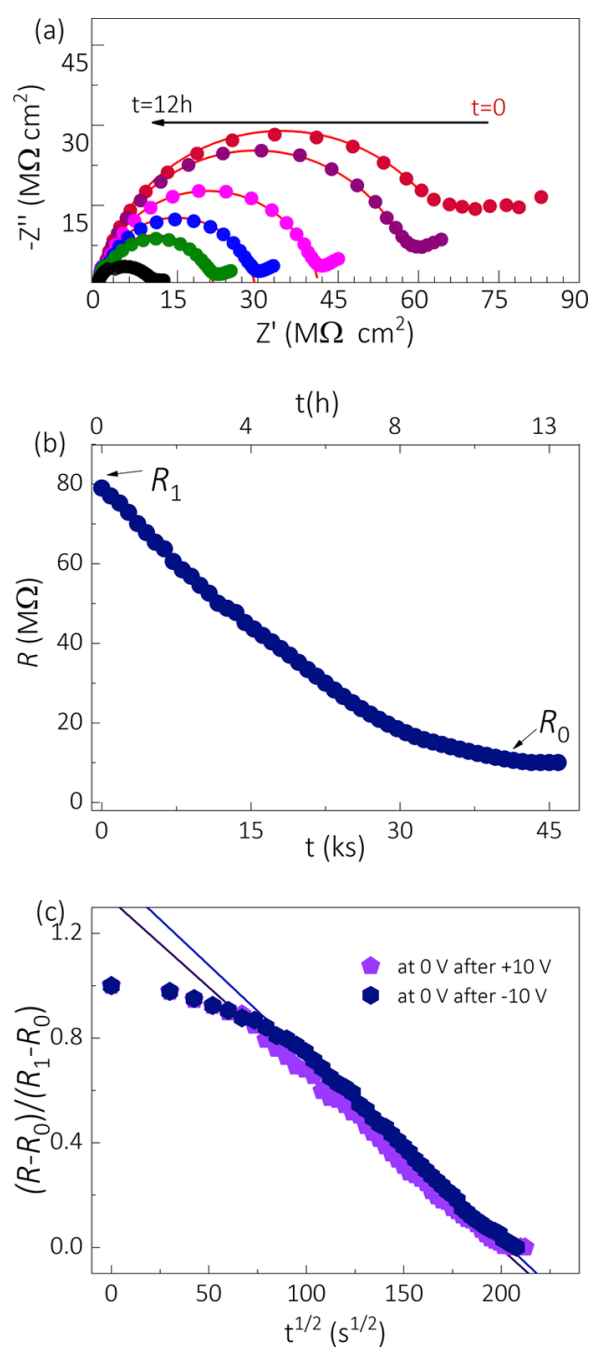

Figure 3. (a) Variation of the impedance at zero bias after removing $+10 \mathrm{~V}$ bias and evolution with time. The dots correspond to the experimental data obtained at different time instants: $0 \mathrm{~h}(\mathrm{red}),+2 \mathrm{~h}$ (purple), $+5 \mathrm{~h}$ (pink), $+7 \mathrm{~h}$ (blue), $+9 \mathrm{~h}$ (green), and $12 \mathrm{~h}$ (black). The red solid lines correspond to the fittings to the equivalent circuit in Figure S2. (b) Long-time relaxation experiments ( $\sim 12 \mathrm{~h})$ showing the variation of the sample resistance extracted from impedance spectral analysis as a function of time measured at zero bias. A transition between initial large resistance $R_{1}$ down to small resistance $R_{0}$ is observed. (c) Variation of the normalized resistance as a function of time following the relationship of eq 3 , for relaxation experiments performed at zero bias after the previous long-term positive and negative bias. The blue/purple solid lines represent linear fittings.

change in electronic carrier density. The mechanism can be viewed as a sort of the dynamic doping process in which mobile ions act as dopants able to locally alter the doping density. An alternative explanation of the resistance variation of Figure $3 \mathrm{~b}$ (not explored here) would imply a hypothetical iondriven modulation of the electronic mobility.

A model of the dynamic doping process accounting for the resistance variation is outlined in Figure S5 of Supporting Information. Our approach simplifies the complex situation by considering a sharp front separating two zones of high and low carrier densities that shift as the ions diffuse to recover the equilibrium ion concentration (background electronic conduction state). A simple expression is thereby derived as 


$$
\frac{R-R_{0}}{R_{1}-R_{0}}=1-\frac{3 \sqrt{2 D_{\text {ion }} t}}{L}
$$

which assumes a one-dimensional diffusion process with Gaussian ion distribution and predicts a time dependence of the kind $\sqrt{t}$, keeping then the diffusive character as previously observed for ionic depolarization currents in Figure 2. Therefore, the resistance in Figure $3 \mathrm{~b}$ can be normalized as $\left(R-R_{0}\right) /\left(R_{1}-R_{0}\right)$ and plotted as a function of $\sqrt{t}$, as presented in Figure 3c. By examining this plot, a linear trend is clearly obeyed with a slope including the ion diffusivity as $3 \sqrt{2 D_{\text {ion }}} / L$, as derived from the analytical model of eq 3 and purposely outlined in Supporting Information. After resistance normalization, very similar trends are reproduced independently of the voltage sign in Figure $3 \mathrm{c}$. The kinetic independence on the bias sign would indicate a bulk origin, rather than interfacial, for the mechanism behind the observed electrical response. We note that a deviation from the linear behavior occurs at shorter times $\left(t<5 \times 10^{3} \mathrm{~s}\right)$ in Figure $3 \mathrm{c}$, for which the simplicity of the model disregards second-order depolarization effects for greater moving ion concentrations. For shorter times, the driving force for ion displacement should certainly incorporate other mechanisms as the relaxation of the ionic built-in voltage should include driftdriven processes in addition to the simple diffusion, in such a way that the $\sqrt{t}$ dependence is no longer obeyed. In any case, the linear response part in Figure $3 c$ seems to reinforce the predominance of diffusion mechanisms for $t>5 \times 10^{3} \mathrm{~s}$ of the short-circuit regime after polarization.

From the linear fitting slope in Figure $3 c$ (valid for longer times) and the experimental parameter $L=1.1 \mathrm{~mm}$, one can obtain a value for the ion diffusion coefficient that results in $D_{\text {ion }}=(3 \pm 1) \times 10^{-8} \mathrm{~cm}^{2} \mathrm{~s}^{-1}$, averaging over several relaxation experiments. We remark that the order of magnitude obtained for $D_{\text {ion }}$ is in agreement with values reported for the diffusion coefficient of iodine-related defects. By nuclear magnetic resonance (NMR), ${ }^{52,53}$ values of order $10^{-9} \mathrm{~cm}^{2} \mathrm{~s}^{-1}$ were obtained for $\mathrm{I}^{-}$, while $\mathrm{MA}^{+}$exhibited smaller values in the range of $10^{-15}$ to $10^{-12} \mathrm{~cm}^{2} \mathrm{~s}^{-1}$. From drift current accompanied by electrical switching of luminescence, ${ }^{34} \mathrm{I}^{-}$ diffusivity is obtained to be around $5 \times 10^{-8}$ to $6 \times 10^{-9}$ $\mathrm{cm}^{2} \mathrm{~s}^{-1}$. Also, current transient ${ }^{54}$ and capacitance transient ${ }^{55}$ analyses gave rise to a diffusion coefficient of order $10^{-8}$ to $10^{-9} \mathrm{~cm}^{2} \mathrm{~s}^{-1}$ for $\mathrm{I}^{-}$. In addition, impedance analysis of the lowfrequency diffusion patterns yielded similar values. ${ }^{49}$ Therefore, we also assume that the relatively fast ion diffusion coefficient encountered here $D_{\text {ion }} \sim 10^{-8} \mathrm{~cm}^{2} \mathrm{~s}^{-1}$ should be related to the transport of mobile iodine defects. Other possible moving ions such as $\mathrm{MA}^{+}$and $\mathrm{Pb}^{2+}$ should certainly exhibit much lower values. $^{48,56}$

As a consequence, it is inferred that iodine vacancies $\mathrm{V}_{\mathrm{I}}^{+}$ dominate and establish the general background doping character. As halide vacancies are considered a shallow donor both for iodide and bromide perovskites, ${ }^{23,57}$ an $n$-type doping character should be larger in zones in which the iodine vacancy concentration increases. Upon biasing, the formation of rich zones of iodine vacancies near the negatively biased contact is expected, and the contrary for the positive contact. Large part of the layer bulk undergoes a dedoping process as dopant ions accumulate at the contacts. Therefore, an internal doping profile is formed that implies that the perovskite bulk can be modeled as a sort of the semiconductor homojunction with a mobile boundary between lower- and higher-doped regions in accordance with the model outlined in Supporting Information. This is what we denote as the dynamic doping process.

\section{CONCLUSIONS}

Summarizing our results, we indicate that the present study reveals several features to be included into a simulation of the general device operation of mm-thick perovskite layers. (1) On the one hand, long-timescale diffusion patterns are encountered which follows a $\sqrt{t}$-dependence. This unambiguously signals the fingerprint of diffusive transport. (2) This time dependence is simultaneously obeyed by current relaxation (Figure 2) and impedance recovering (Figure 3). While longtime negative current undershoot at zero bias exclusively registers the ion displacement, the impedance captures the electronic small perturbation response at different depolarization stages. This independent observation of diffusion patterns reinforces the validity of our explanation. (3) As a consequence, an ionic-electronic interplay should exist between mobile ions that act as dopants and electronic carriers. The electronic doping profile changes depending on the actual ion bulk distribution. This is the essence of the dynamic doping mechanism formulated here. (4) The kinetics of ionic depolarization is a multistep process. It starts by an initial collapse of the ion diffusion layer $\left(\tau_{\text {diff }} \sim 200 \mathrm{~s}\right)$, built up after biasing. Afterward, long-range diffusion occurs involving much longer times $\left(\tau_{\mathrm{L}} \sim 10^{5} \mathrm{~s}\right)$. From the analysis, the ion diffusion coefficient is extracted which lies within the range of $10^{-8} \mathrm{~cm}^{2} \mathrm{~s}^{-1}$, presumable connected to the iodine defect transport.

We have proposed here a connection between ionic and electronic properties that allow progressing into the halide perovskite device physics and operating modes. Technological realizations such as solar cells, light-emitting diodes, and, particularly, X- and $\gamma$-ray detectors would benefit from this fundamental information, as far as long-timescale functioning and current stabilization under biasing are concerned.

\section{EXPERIMENTAL SECTION}

Sample Preparation. The samples under consideration were $\sim 1$ $\mathrm{mm}$-thick MAPI microcrystalline pellets with a diameter of $15 \mathrm{~mm}$. The pellets were made by a soft-sintering process, which is described in detail in ref 7 . The microcrystalline MAPI powder is commercially available from Xi'an Polymer Light Technology Corp. It was filled into a powder container with adjustable height, and a polished cylinder was placed above it. The hydraulic press (PerkinElmer) applies a pressure of $55 \mathrm{MPa}$ (1t) for $30 \mathrm{~min}$ at $70{ }^{\circ} \mathrm{C}$. On the resulting pellet, two electrodes ( $\mathrm{Pt}$ and $\mathrm{Cr}$ ) with a thickness of around $100 \mathrm{~nm}$ were deposited via physical vapor deposition. Their active area was $1 \mathrm{~cm}^{2}$.

Structural and Optical Characterization Techniques. Through measurements of scanning electron microscopy, the polycrystalline nature of the perovskite pellet is observed (Figure $\mathrm{S6a}$ ). The X-ray diffraction pattern (Figure S6b) shows the most prominent peaks with the (110), (220), and (310) peaks at 14.1, 28.4, and $32.1^{\circ}$, respectively. The peaks indicate the tetragonal $\mathrm{MAPbI}_{3}$ phase with an $I 4 \mathrm{~cm}$ space group. These values are in good agreement with comparative XRD data of a typical MAPI film. ${ }^{58,59}$

Electrical Measurements. The chronoamperometry experiments and the impedance spectroscopy (IS) characterizations were carried out with a PGSTAT302N potentiostat from Metrohm AUTOLAB. Measurements of current and impedance were carried out under several long-time DC bias conditions in the range of \pm 10 to $0 \mathrm{~V}$. The samples were kept in the dark at $295 \mathrm{~K}$ with $\mathrm{N}_{2}$ circulation in order to prevent from humidity- or oxygen-induced degradation. 


\section{ASSOCIATED CONTENT}

\section{(3) Supporting Information}

The Supporting Information is available free of charge at https://pubs.acs.org/doi/10.1021/acsami.1c06046.

Sample preparation and characterization, general current transients and impedance analysis, and model for resistance relaxation $(\mathrm{PDF})$

\section{AUTHOR INFORMATION}

Corresponding Author

Germà Garcia-Belmonte - Institute of Advanced Materials (INAM), Universitat Jaume I, Castelló ES12006, Spain; (ㄱ orcid.org/0000-0002-0172-6175; Email: garciag@uji.es

\section{Authors}

Marisé García-Batlle - Institute of Advanced Materials (INAM), Universitat Jaume I, Castelló ES12006, Spain

Sarah Deumel - Siemens Healthineers AG, Erlangen 91058, Germany; Institute-Materials for Electronics and Energy Technology (i-MEET), Department of Materials Science and Engineering, Friedrich-Alexander-Universitaet ErlangenNuernberg, Nuernberg 90429, Germany

Judith E. Huerdler - Siemens Healthineers AG, Erlangen 91058, Germany

Sandro F. Tedde - Siemens Healthineers AG, Erlangen 91058, Germany; 이이이.org/0000-0002-6797-9570

Antonio Guerrero - Institute of Advanced Materials (INAM), Universitat Jaume I, Castelló ES12006, Spain; (1) orcid.org/0000-0001-8602-1248

Osbel Almora - Institute of Advanced Materials (INAM), Universitat Jaume I, Castelló ES12006, Spain

Complete contact information is available at: https://pubs.acs.org/10.1021/acsami.1c06046

\section{Notes}

The authors declare no competing financial interest.

\section{ACKNOWLEDGMENTS}

This work has received funding from the European Union's Horizon 2020 research and innovation program under the Photonics Public Private Partnership (www.photonics21.org) with the project PEROXIS under the grant agreement $\mathrm{N}^{\circ}$ 871336. M.G.-B. acknowledges Generalitat Valenciana for a grant (number GRISOLIAP/2018/073).

\section{REFERENCES}

(1) Green, M. A.; Dunlop, E. D.; Hohl-Ebinger, J.; Yoshita, M.; Kopidakis, N.; Hao, X. Solar Cell Efficiency Tables (Version 56). Prog. Photovoltaics Res. Appl. 2020, 28, 629-638.

(2) Heiss, W.; Brabec, C. Perovskites Target X-Ray Detection. Nat. Photonics 2016, 10, 288-289.

(3) Wei, H.; Fang, Y.; Mulligan, P.; Chuirazzi, W.; Fang, H.-H.; Wang, C.; Ecker, B. R.; Gao, Y.; Loi, M. A.; Cao, L.; Huang, J. Sensitive X-Ray Detectors Made of Methylammonium Lead Tribromide Perovskite Single Crystals. Nat. Photonics 2016, 10, 333-339.

(4) Murali, B.; Kolli, H. K.; Yin, J.; Ketavath, R.; Bakr, O. M.; Mohammed, O. F. Single Crystals: The Next Big Wave of Perovskite Optoelectronics. ACS Mater. Lett. 2020, 2, 184-214.

(5) He, Y.; Matei, L.; Jung, H. J.; McCall, K. M.; Chen, M.; Stoumpos, C. C.; Liu, Z.; Peters, J. A.; Chung, D. Y.; Wessels, B. W.; Wasielewski, M. R.; Dravid, V. P.; Burger, A.; Kanatzidis, M. G. High spectral resolution of gamma-rays at room temperature by perovskite CsPbBr3 single crystals. Nat. Commun. 2018, 9, 1609.

(6) He, Y.; Petryk, M.; Liu, Z.; Chica, D. G.; Hadar, I.; Leak, C.; Ke, W.; Spanopoulos, I.; Lin, W.; Chung, D. Y.; Wessels, B. W.; He, Z.; Kanatzidis, M. G. $\mathrm{CsPbBr}_{3}$ Perovskite Detectors with $1.4 \%$ Energy Resolution for High-Energy $\gamma$-Rays. Nat. Photonics 2021, 15, 36-42.

(7) Deumel, S.; Breemen, A. v.; Gelinck, G.; Peeters, B.; Maas, J.; Verbeek, R.; Akkerman, H.; Meulenkamp, E.; Huerdler, J. E.; Acharya, M.; García-Batlle, M.; Almora, O.; Guerrero, A.; Garcia-Belmonte, G.; Heiss, W.; Schmidt, O.; Tedde, S. F. Sub-Nanogray X-Ray Imaging with Soft-sintered Methylammonium Lead Triiodide Perovskites. Nat. Electron. 2021 submitted.

(8) Motta, C.; El-Mellouhi, F.; Sanvito, S. Charge Carrier Mobility in Hybrid Halide Perovskites. Sci. Rep. 2015, 5, 12746.

(9) Herz, L. M. Charge-Carrier Mobilities in Metal Halide Perovskites: Fundamental Mechanisms and Limits. ACS Energy Lett. 2017, 2, 1539-1548.

(10) Duijnstee, E. A.; Le Corre, V. M.; Johnston, M. B.; Koster, L. J. A.; Lim, J.; Snaith, H. J. Understanding Dark Current-Voltage Characteristics in Metal-Halide Perovskite Single Crystals. Phys. Rev. Appl. 2021, 15, 014006.

(11) Takada, J.; Yamaguchi, M.; Fukada, N.; Nishimura, K.; Tawada, Y. Thermal Degradation of a-Si:H Solar Cells by Dopant Diffusion. Jpn. J. Appl. Phys. 1987, 26, 889-892.

(12) Feldmann, F.; Schön, J.; Niess, J.; Lerch, W.; Hermle, M. Studying Dopant Diffusion from Poly-Si Passivating Contacts. Sol. Energy Mater. Sol. Cells 2019, 200, 109978.

(13) Schmidt, J. Light-Induced Degradation in Crystalline Silicon Solar Cells. Solid State Phenom. 2003, 95-96, 187-196.

(14) Adey, J.; Jones, R.; Palmer, D. W.; Briddon, P. R.; Öberg, S. Degradation of Boron-Doped Czochralski-Grown Silicon Solar Cells. Phys. Rev. Lett. 2004, 93, 055504.

(15) van Reenen, S.; Janssen, R. A. J.; Kemerink, M. Doping Dynamics in Light-Emitting Electrochemical Cells. Org. Electron. 2011, 12, 1746-1753.

(16) Lenes, M.; Garcia-Belmonte, G.; Tordera, D.; Pertegás, A.; Bisquert, J.; Bolink, H. J. Operating Modes of Sandwiched LightEmitting Electrochemical Cells. Adv. Funct. Mater. 2011, 21, 15811586.

(17) Brütting, W.; Adachi, C. Physics of Organic Semiconductors; Wiley-VCH: Weinheim, 2012.

(18) Gunkel, F.; Christensen, D. V.; Chen, Y. Z.; Pryds, N. Oxygen Vacancies: The (In)visible Friend of Oxide Electronics. Appl. Phys. Lett. 2020, 116, 120505.

(19) Waser, R.; Dittmann, R.; Staikov, G.; Szot, K. Redox-Based Resistive Switching Memories - Nanoionic Mechanisms, Prospects, and Challenges. Adv. Mater. 2009, 21, 2632-2663.

(20) Almora, O.; Lopez-Varo, P.; Cho, K. T.; Aghazada, S.; Meng, W.; Hou, Y.; Echeverría-Arrondo, C.; Zimmermann, I.; Matt, G. J.; Jiménez-Tejada, J. A.; Brabec, C. J.; Nazeeruddin, M. K.; GarciaBelmonte, G. Ionic Dipolar Switching Hinders Charge Collection in Perovskite Solar Cells with Normal and Inverted Hysteresis. Sol. Energy Mater. Sol. Cells 2019, 195, 291-298.

(21) Bertoluzzi, L.; Boyd, C. C.; Rolston, N.; Xu, J.; Prasanna, R.; O'Regan, B. C.; McGehee, M. D. Mobile Ion Concentration Measurement and Open-Access Band Diagram Simulation Platform for Halide Perovskite Solar Cells. Joule 2020, 4, 109-127.

(22) Buin, A.; Comin, R.; Xu, J.; Ip, A. H.; Sargent, E. H. HalideDependent Electronic Structure of Organolead Perovskite Materials. Chem. Mater. 2015, 27, 4405-4412.

(23) Yin, W.-J.; Shi, T.; Yan, Y. Unusual defect physics in CH3NH3PbI3 perovskite solar cell absorber. Appl. Phys. Lett. 2014, 104, 063903.

(24) Azpiroz, J. M.; Mosconi, E.; Bisquert, J.; De Angelis, F. Defect Migration in Methylammonium Lead Iodide and its Role in Perovskite Solar Cell Operation. Energy Environ. Sci. 2015, 8, $2118-2127$. 
(25) Yang, D.; Ming, W.; Shi, H.; Zhang, L.; Du, M.-H. Fast Diffusion of Native Defects and Impurities in Perovskite Solar Cell Material CH3NH3PbI3. Chem. Mater. 2016, 28, 4349-4357.

(26) Bergmann, V. W.; Guo, Y.; Tanaka, H.; Hermes, I. M.; Li, D.; Klasen, A.; Bretschneider, S. A.; Nakamura, E.; Berger, R.; Weber, S. A. L. Local Time-Dependent Charging in a Perovskite Solar Cell. ACS Appl. Mater. Interfaces 2016, 8, 19402-19409.

(27) Ahmadi, M.; Collins, L.; Higgins, K.; Kim, D.; Lukosi, E.; Kalinin, S. V. Spatially Resolved Carrier Dynamics at MAPbBr3 Single Crystal-Electrode Interface. ACS Appl. Mater. Interfaces 2019, 11, 41551-41560.

(28) Zhu, T.-Y.; Shu, D.-J. Role of Ionic Charge Accumulation in Perovskite Solar Cell: Carrier Transfer in Bulk and Extraction at Interface. J. Phys. Chem. C 2019, 123, 5312-5320.

(29) Pospisil, J.; Guerrero, A.; Zmeskal, O.; Weiter, M.; Gallardo, J. J.; Navas, J.; Garcia-Belmonte, G. Reversible Formation of Gold Halides in Single-Crystal Hybrid-Perovskite/Au Interface upon Biasing and Effect on Electronic Carrier Injection. Adv. Funct. Mater. 2019, 29, 1900881.

(30) Wang, J.; Senanayak, S. P.; Liu, J.; Hu, Y.; Shi, Y.; Li, Z.; Zhang, C.; Yang, B.; Jiang, L.; Di, D.; Ievlev, A. V.; Ovchinnikova, O. S.; Ding, T.; Deng, H.; Tang, L.; Guo, Y.; Wang, J.; Xiao, K.; Venkateshvaran, D.; Jiang, L.; Zhu, D.; Sirringhaus, H. Investigation of Electrode Electrochemical Reactions in $\mathrm{CH} 3 \mathrm{NH} 3 \mathrm{PbBr} 3$ Perovskite SingleCrystal Field-Effect Transistors. Adv. Mater. 2019, 31, 1902618.

(31) Pockett, A.; Eperon, G. E.; Sakai, N.; Snaith, H. J.; Peter, L. M.; Cameron, P. J. Microseconds, Milliseconds and Seconds: Deconvoluting the Dynamic Behaviour of Planar Perovskite Solar Cells. Phys. Chem. Chem. Phys. 2017, 19, 5959-5970.

(32) Yang, C.; El-Demellawi, J. K.; Yin, J.; Velusamy, D. B.; Emwas, A.-H. M.; El-Zohry, A. M.; Gereige, I.; AlSaggaf, A.; Bakr, O. M.; Alshareef, H. N.; Mohammed, O. F. MAPbI3 Single Crystals Free from Hole-Trapping Centers for Enhanced Photodetectivity. ACS Energy Lett. 2019, 4, 2579-2584.

(33) Fischer, M.; Tvingstedt, K.; Baumann, A.; Dyakonov, V. Doping Profile in Planar Hybrid Perovskite Solar Cells Identifying Mobile Ions. ACS Appl. Energy Mater. 2018, 1, 5129-5134.

(34) Li, C.; Guerrero, A.; Huettner, S.; Bisquert, J. Unravelling the Role of Vacancies in Lead Halide Perovskite through Electrical Switching of Photoluminescence. Nat. Commun. 2018, 9, 5113.

(35) García-Batlle, M.; Baussens, O.; Amari, S.; Zaccaro, J.; GrosDaillon, E.; Verilhac, J.-M.; Guerrero, A.; Garcia-Belmonte, G. Moving Ions Vary Electronic Conductivity in Lead Bromide Perovskite Single Crystals through Dynamic Doping. Adv. Electron. Mater. 2020, 6, 2000485.

(36) Wang, H.; Guerrero, A.; Bou, A.; Al-Mayouf, A. M.; Bisquert, J. Kinetic and Material Properties of Interfaces Governing Slow Response and Long Timescale Phenomena in Perovskite Solar Cells. Energy Environ. Sci. 2019, 12, 2054-2079.

(37) Liu, J.; Hu, M.; Dai, Z.; Que, W.; Padture, N. P.; Zhou, Y. Correlations between Electrochemical Ion Migration and Anomalous Device Behaviors in Perovskite Solar Cells. ACS Energy Lett. 2021, 6, $1003-1014$.

(38) Lopez-Varo, P.; Jiménez-Tejada, J. A.; García-Rosell, M.; Ravishankar, S.; Garcia-Belmonte, G.; Bisquert, J.; Almora, O. Device Physics of Hybrid Perovskite Solar Cells: Theory and Experiment. Adv. Energy Mater. 2018, 8, 1702772.

(39) Kerner, R. A.; Rand, B. P. Ionic-Electronic Ambipolar Transport in Metal Halide Perovskites: Can Electronic Conductivity Limit Ionic Diffusion? J. Phys. Chem. Lett. 2018, 9, 132-137.

(40) Reichert, S.; An, Q.; Woo, Y.-W.; Walsh, A.; Vaynzof, Y.; Deibel, C. Probing the Ionic Defect Landscape in Halide Perovskite Solar Cells. Nat. Commun. 2020, 11, 6098.

(41) Moia, D.; Gelmetti, I.; Calado, P.; Fisher, W.; Stringer, M.; Game, O.; Hu, Y.; Docampo, P.; Lidzey, D.; Palomares, E.; Nelson, J.; Barnes, P. R. F. Ionic-to-Electronic Current Amplification in Hybrid Perovskite Solar Cells: Ionically Gated Transistor-Interface Circuit Model Explains Hysteresis and Impedance of Mixed Conducting Devices. Energy Environ. Sci. 2019, 12, 1296-1308.
(42) Li, D.; Wu, H.; Cheng, H.-C.; Wang, G.; Huang, Y.; Duan, X. Electronic and Ionic Transport Dynamics in Organolead Halide Perovskites. ACS Nano 2016, 10, 6933-6941.

(43) Xiao, Z.; Yuan, Y.; Shao, Y.; Wang, Q.; Dong, Q.; Bi, C.; Sharma, P.; Gruverman, A.; Huang, J. Giant Switchable Photovoltaic Effect in Organometal Trihalide Perovskite Devices. Nat. Mater. 2015, 14, 193-198.

(44) Zhao, Y.; Liang, C.; Zhang, H.; Li, D.; Tian, D.; Li, G.; Jing, X.; Zhang, W.; Xiao, W.; Liu, Q.; Zhang, F.; He, Z. Anomalously large interface charge in polarity-switchable photovoltaic devices: an indication of mobile ions in organic-inorganic halide perovskites. Energy Environ. Sci. 2015, 8, 1256-1260.

(45) Eames, C.; Frost, J. M.; Barnes, P. R. F.; O’Regan, B. C.; Walsh, A.; Islam, M. S. Ionic Transport in Hybrid Lead Iodide Perovskite Solar Cells. Nat. Commun. 2015, 6, 7497.

(46) Bag, M.; Renna, L. A.; Adhikari, R. Y.; Karak, S.; Liu, F.; Lahti, P. M.; Russell, T. P.; Tuominen, M. T.; Venkataraman, D. Kinetics of Ion Transport in Perovskite Active Layers and Its Implications for Active Layer Stability. J. Am. Chem. Soc. 2015, 137, 13130-13137.

(47) Richardson, G.; O’Kane, S. E. J.; Niemann, R. G.; Peltola, T. A.; Foster, J. M.; Cameron, P. J.; Walker, A. B. Can slow-moving ions explain hysteresis in the current-voltage curves of perovskite solar cells? Energy Environ. Sci. 2016, 9, 1476-1485.

(48) Yang, T.-Y.; Gregori, G.; Pellet, N.; Grätzel, M.; Maier, J. The Significance of Ion Conduction in a Hybrid Organic-Inorganic LeadIodide-Based Perovskite Photosensitizer. Angew. Chem., Int. Ed. 2015, 54, 7905-7910.

(49) Peng, W.; Aranda, C.; Bakr, O. M.; Garcia-Belmonte, G.; Bisquert, J.; Guerrero, A. Quantification of Ionic Diffusion in Lead Halide Perovskite Single Crystals. ACS Energy Lett. 2018, 3, 14771481.

(50) Savenije, T. J.; Ponseca, C. S.; Kunneman, L.; Abdellah, M.; Zheng, K.; Tian, Y.; Zhu, Q.; Canton, S. E.; Scheblykin, I. G.; Pullerits, T.; Yartsev, A.; Sundström, V. Thermally Activated Exciton Dissociation and Recombination Control the Carrier Dynamics in Organometal Halide Perovskite. J. Phys. Chem. Lett. 2014, 5, 21892194.

(51) Almora, O.; Garcia-Belmonte, G. Light Capacitances in Silicon and Perovskite Solar Cells. Sol. Energy 2019, 189, 103-110.

(52) Senocrate, A.; Moudrakovski, I.; Acartürk, T.; Merkle, R.; Kim, G. Y.; Starke, U.; Grätzel, M.; Maier, J. Slow CH3NH3+ Diffusion in $\mathrm{CH} 3 \mathrm{NH} 3 \mathrm{PbI} 3$ under Light Measured by Solid-State NMR and Tracer Diffusion. J. Phys. Chem. C 2018, 122, 21803-21806.

(53) Senocrate, A.; Moudrakovski, I.; Kim, G. Y.; Yang, T.-Y.; Gregori, G.; Grätzel, M.; Maier, J. The Nature of Ion Conduction in Methylammonium Lead Iodide: A Multimethod Approach. Angew. Chem., Int. Ed. 2017, 56, 7755-7759.

(54) Bertoluzzi, L.; Belisle, R. A.; Bush, K. A.; Cheacharoen, R.; McGehee, M. D.; O’Regan, B. C. In Situ Measurement of ElectricField Screening in Hysteresis-Free PTAA/FA0.83Cs0.17Pb(I0.83Br0.17)3/C60 Perovskite Solar Cells Gives an Ion Mobility of $\sim 3 \times 10-7 \mathrm{~cm} 2 /(\mathrm{V} \mathrm{s}), 2$ Orders of Magnitude Faster than Reported for Metal-Oxide-Contacted Perovskite Cells with Hysteresis. J. Am. Chem. Soc. 2018, 140, 12775-12784.

(55) Futscher, M. H.; Lee, J. M.; McGovern, L.; Muscarella, L. A.; Wang, T.; Haider, M. I.; Fakharuddin, A.; Schmidt-Mende, L.; Ehrler, B. Quantification of ion migration in $\mathrm{CH} 3 \mathrm{NH} 3 \mathrm{PbI} 3$ perovskite solar cells by transient capacitance measurements. Mater. Horiz. 2019, 6, 1497-1503.

(56) Weber, S. A. L.; Hermes, I. M.; Turren-Cruz, S.-H.; Gort, C.; Bergmann, V. W.; Gilson, L.; Hagfeldt, A.; Graetzel, M.; Tress, W.; Berger, R. How the Formation of Interfacial Charge Causes Hysteresis in Perovskite Solar Cells. Energy Environ. Sci. 2018, 11, 2404-2413.

(57) Motti, S. G.; Meggiolaro, D.; Martani, S.; Sorrentino, R.; Barker, A. J.; De Angelis, F.; Petrozza, A. Defect Activity in Lead Halide Perovskites. Adv. Mater. 2019, 31, 1901183.

(58) Phillips, L. J.; Rashed, A. M.; Treharne, R. E.; Kay, J.; Yates, P.; Mitrovic, I. Z.; Weerakkody, A.; Hall, S.; Durose, K. Maximizing the optical performance of planar $\mathrm{CH} 3 \mathrm{NH} 3 \mathrm{PbI} 3$ hybrid perovskite 
heterojunction stacks. Sol. Energy Mater. Sol. Cells 2016, 147, 327333.

(59) Aranda, C.; Cristobal, C.; Shooshtari, L.; Li, C.; Huettner, S.; Guerrero, A. Formation Criteria of High Efficiency Perovskite Solar Cells under Ambient Conditions. Sustainable Energy Fuels 2017, 1, 540-547. 